\title{
VOLUME DENSITIES WITH THE MEAN VALUE PROPERTY FOR HARMONIC FUNCTIONS
}

\author{
W. HANSEN AND I. NETUKA
}

(Communicated by J. Marshall Ash)

\begin{abstract}
On a bounded domain $U$ in $\mathbb{R}^{d}$ containing the origin, probability measures $\mu$ which have a density $w$ with respect to Lebesgue measure and satisfy $h(0)=\int h d \mu$ for every bounded harmonic function on $U$ are studied. A domain $U$ is constructed such that $\inf w(U)=0$ for any such measure. (This solves a problem proposed by A. Cornea.) If, however, $U$ has smooth boundary, then $\mu$ having a density $w \in \mathscr{C}^{\infty}(U)$ which is bounded away from zero on $U$ can be constructed. On the other hand, for arbitrary $U$ it is always possible to choose a strictly positive $w \in \mathscr{C}^{\infty}(U)$ tending to zero at $\partial U$.
\end{abstract}

\section{INTRODUCTION}

Let $U$ be a bounded domain in $\mathbb{R}^{d}, d \geq 2$, and $0 \in U$. There are many positive measures $\mu$ on $U$ such that $\mu(U)>0$ and

$$
h(0)=\frac{1}{\mu(U)} \int_{U} h d \mu
$$

holds for every $h \in \mathscr{H}_{b}(U)$, the set of bounded harmonic functions on $U$. For various purposes, such measures were investigated, e.g., in [CD, Fl, FL, Ga, $\mathrm{Hi}$, $\mathrm{Sm}, \mathrm{Za}]$.

If desired, $\mu$ can be chosen to be absolutely continuous with respect to Lebesgue measure $\lambda$ on $U$, say, $\mu=w \lambda$. (Indeed, take, e.g., $w=1 / \lambda(B)$ on a ball $B$ contained in $U$ and $w=0$ elsewhere in $U$.)

In a discussion during the International Conference on Potential Theory (Nagoya, 1990), A. Cornea raised the problem whether there always exists a function $w$ fulfilling $(*)$ that is bounded away from zero on $U$.

A counterexample constructed in $\S 2$ of this paper provides a negative solution to this problem. Our domain $U$ is regular for the Dirichlet problem, and, actually, its boundary behaves badly near a single point only. It turns out that the problem is related to the integrability of positive harmonic functions on $U$ with respect to $\lambda$.

Section 3 contains positive results: There always exists a strictly positive $w \in$ $\mathscr{C}^{\infty}(U)$ satisfying $(*)$. If, moreover, the boundary of $U$ is smooth enough

Received by the editors August 25, 1992 and, in revised form, April 8, 1993.

1991 Mathematics Subject Classification. Primary 31A05, 31B05.

Key words and phrases. Harmonic functions, mean value property, balayage measures. 
(e.g., of class $\mathscr{C}^{1+\varepsilon}$ ), then a function $w \in \mathscr{C}^{\infty}(U)$ that is bounded away from zero can be produced. The latter result is based on [Wi].

\section{A COUNTEREXAMPLE}

For $z \in \mathbb{R}^{d}$ and $r>0$ denote $B_{r}(z)=\left\{z^{\prime} \in \mathbb{R}^{d}:\left\|z^{\prime}-z\right\|<r\right\}$. For $n \in \mathbb{N} \cup\{0\}$ let $y_{n}=\left(2^{-n}, 0, \ldots, 0\right), x_{n}=\left(y_{n}+y_{n+1}\right) / 2, r_{n}=2^{-n-2}$,

$$
s_{n}= \begin{cases}\exp \left(-4^{2 n+1}\right) & \text { if } d=2, \\ 4^{-d /(d-2)(n+1)} & \text { if } d>2,\end{cases}
$$

$V_{n}=B_{r_{n}}\left(x_{n}\right), W_{n}=B_{s_{n}}\left(y_{n}\right)$, and

$$
U=\bigcup_{n=0}^{\infty} V_{n} \cup \bigcup_{n=1}^{\infty} W_{n}
$$

Notice that $U$ is a regular domain contained in $B_{1}(0)$.

Denote $H(U)=\left\{h \in \mathscr{C}(\bar{U}):\left.h\right|_{U}\right.$ harmonic $\}$, and write $H^{+}(U)$ for the set of positive functions from $H(U)$. As usual, for a resolutive numerical function $f$ on $\partial U$, let $H_{U} f$ denote the corresponding solution of the Dirichlet problem.

2.1. Proposition. $\sup \left\{\int_{U} h d \lambda: h \in H^{+}(U), h\left(x_{0}\right) \leq 1\right\}=\infty$.

Proof. Let $B=B_{1}(0), a_{+}=(1,0, \ldots, 0), a_{-}=(-1,0, \ldots, 0)$,

$$
D=B \backslash\left(B_{1 / 2}\left(a_{+}\right) \cup B_{1 / 2}\left(a_{-}\right)\right), \quad L=\partial B \backslash\left(B_{1 / 4}\left(a_{+}\right) \cup B_{1 / 4}\left(a_{-}\right)\right),
$$

and $g=H_{B} 1_{L}$. Denote $\alpha=\inf g(D), \beta=\lambda(D)$. It is easy to see that $\alpha>0$.

In the following considerations sweeping and balayage are understood with respect to (the $\mathscr{P}$-harmonic space) $X=B_{2}(0)$ if $d=2$ and with respect to $X=\mathbb{R}^{d}$ if $d>2$.

Let $x=x_{0}$, and fix $n \in \mathbb{N}$. If $d=2$, then

$$
R_{1}^{W_{n}}(x) \leq \frac{1-\ln \left\|y_{n}-x\right\|}{1-\ln s_{n}}<4\left(\ln \frac{1}{s_{n}}\right)^{-1} \leq 4^{-2 n} .
$$

For $d>2$ we have

$$
R_{1}^{W_{n}}(x)=\frac{s_{n}^{d-2}}{\left\|x-y_{n}\right\|^{d-2}} \leq 4^{-d(n+1)} 4^{d-2}<4^{-d n} .
$$

The measure $\mu_{x}:=\varepsilon_{x}^{C\left(U \backslash \bar{V}_{n}\right)}=\varepsilon_{x}^{C U \cup \bar{V}_{n}}$ is supported by the boundary of the connected component of $U \backslash \bar{V}_{n}$ containing $x$. Defining $A_{n}:=\bar{V}_{n} \cap \bar{W}_{n}$ we hence know that $\mu_{x}\left(\bar{V}_{n}\right)=\mu_{x}\left(A_{n}\right)$. General properties of balayage (see [BH, p. 317]) imply that $\varepsilon_{x}^{C U}\left(\bar{V}_{n}\right) \leq \mu_{x}\left(\bar{V}_{n}\right)$ and $\mu_{x}\left(A_{n}\right) \leq \varepsilon_{x}^{A_{n}}(X)$ (of course, one could just as well use the boundary minimum principle to obtain these inequalities). Since $\varepsilon_{x}^{A_{n}}(X) \leq R_{1}^{A_{n}}(x) \leq R_{1}^{W_{n}}(x) \leq 4^{-d n}$, we conclude that $\varepsilon_{x}^{C U}\left(\bar{V}_{n}\right)<4^{-d n}$. Choose $f_{n} \in \mathscr{C}(\partial U)$ such that $0 \leq f_{n} \leq 4^{d n}, f_{n}=0$ on $C \bar{V}_{n}$, and $f_{n}=4^{d n}$ on $L_{n}=\partial V_{n} \backslash\left(B_{r_{n} / 4}\left(y_{n}\right) \cup B_{r_{n} / 4}\left(y_{n+1}\right)\right)$. Denoting

$$
D_{n}=V_{n} \backslash\left(B_{r_{n} / 2}\left(y_{n}\right) \cup B_{r_{n} / 2}\left(y_{n+1}\right)\right), \quad g_{n}=H_{V_{n}} 1_{L_{n}}
$$

we get (using the invariance properties of harmonic functions)

$$
\int_{D_{n}} g_{n} d \lambda \geq \alpha \beta r_{n}^{d}
$$


Since $H_{U} f_{n} \geq 4^{d n} g_{n}$ on $V_{n}$ by the minimum principle, we obtain that

$$
\int_{U} H_{U} f_{n} d \lambda \geq 4^{d n} \int_{D_{n}} g_{n} d \lambda \geq \alpha \beta 2^{d(n-2)} .
$$

On the other hand, for every $n \in \mathbb{N}$,

$$
H_{U} f_{n}(x) \leq 4^{d n} \varepsilon_{x}^{C U}\left(\bar{V}_{n}\right) \leq 1,
$$

finishing the proof.

Obviously, (2.1) implies the existence of a harmonic function $h \geq 0$ on $U$ which is not integrable (it suffices to choose $h_{n} \in H^{+}(U)$ such that $h_{n}\left(x_{0}\right)=1$, $\int_{U} h_{n} d \lambda \geq 2^{n}$, and to take $\left.h=\sum_{n=1}^{\infty} 2^{-n} h_{n}\right)$. In fact, our proof yields a stronger result:

2.2. Proposition. There exists $f: \partial U \rightarrow[0, \infty]$ continuous (and finite) on $\partial U \backslash\{0\}$ such that $H_{U} f\left(x_{0}\right) \leq 1$ and $\int_{U} H_{U} f d \lambda=\infty$.

Proof. Define $f_{n}$ as above and take $f=\sum_{n=1}^{\infty} 2^{-n} f_{n}$.

Moreover, it is an immediate consequence of $(2.1)$ that we have constructed a counterexample to the question of $\mathrm{A}$. Cornea:

2.3. Corollary. Let $\nu$ be a finite positive measure on $U$ and $a \geq 0$, and assume that $\int_{U} h d(a \lambda+\nu)=h\left(x_{0}\right)$ for all $h \in H(U)$. Then $a=0$.

Proof. Let $h \in H^{+}(U), h\left(x_{0}\right) \leq 1$. By assumption,

$$
a \int_{U} h d \lambda \leq \int_{U} h d(a \lambda+\nu) \leq 1,
$$

hence $a=0$ by $(2.1)$.

\section{STRICTLY POSITIVE DENSITIES}

3.1. Lemma. Let $U \subset \mathbb{R}^{d}$ be a bounded domain, $0 \in U$. Then there exists a family $\left(U_{\alpha}\right)_{\alpha>0}$ of regular subdomains of $U$ such that $\bar{U}_{\beta} \subset U_{\alpha}$ for all $0<\alpha<$ $\beta<\infty, \bigcap_{\alpha>0} U_{\alpha}=\{0\}, \bigcup_{\alpha>0} \partial U_{\alpha}=U \backslash\{0\}$.

Proof. If $U$ is regular, it suffices to consider $g=G_{U}(0, \cdot) \quad\left(G_{U}\right.$ being the Green function for $U$ ) and to define $U_{\alpha}=\{g>\alpha\}$. Indeed, since $\lim _{x \rightarrow z} g(x)$ $=0$ for every $z \in \partial U$, we know that $\partial U_{\alpha} \subset U, g=\alpha$ on $\partial U_{\alpha}$. In particular, $U_{\alpha}$ is regular, since $g-\alpha$ is a barrier for the points of $\partial U_{\alpha}$. If $W$ were a connected component of $U_{\alpha}$ not containing 0 then $g=\alpha$ on $\partial W$, hence $g=\alpha$ on $W$ by the minimum principle, thus $W \cap U_{\alpha}=\varnothing$, a contradiction. So $U_{\alpha}$ is connected.

In the general case we take an open ball $W_{0}=B_{r}(0)$ such that $\bar{W}_{0} \subset U$ and define

$$
U_{\alpha}=B_{r / \alpha}(0), \quad \alpha>1 .
$$

Moreover, we choose an increasing sequence $\left(W_{j}\right)$ of domains in $U$ such that $\bar{W}_{j} \subset W_{j+1}$ and $W_{j+1} \backslash \bar{W}_{j}$ is regular for all $j \geq 0, \bigcup_{j=0}^{\infty} W_{j}=U$, and define

$$
U_{t}= \begin{cases}W_{j}, & t=2^{-j}, j=0,1,2, \ldots, \\ \bar{W}_{j} \cup\left\{H_{W_{j+1} \backslash \bar{W}_{j}} 1_{\partial W_{j}}>2^{j+1} t-1\right\}, & 2^{-(j+1)}<t<2^{-j}, j=0,1,2, \ldots\end{cases}
$$


Arguing similarly as before it is easily verified that the family $\left(U_{\alpha}\right)_{\alpha>0}$ has the desired properties. (The sequence $\left(W_{j}\right)$ can be obtained in the following way: Take an increasing sequence $\left(K_{n}\right)$ of connected compact sets such that $\bigcup_{n=1}^{\infty} K_{n}=U$. Suppose that $j \in \mathbb{N}$ and a domain $W_{j-1}$ satisfying $0 \in \bar{W}_{j-1} \subset U$ has already been chosen. Take $k \in \mathbb{N}$ such that $2^{-k} \sqrt{d}<$ $\operatorname{dist}\left(K_{j} \cup \bar{W}_{j-1}, C U\right)$, and let $W_{j}$ be the interior of the union of all cubes of the form $\prod_{j=1}^{d}\left[m_{j} 2^{-k},\left(m_{j}+1\right) 2^{-k}\left[, m_{j} \in \mathbb{Z}\right.\right.$, intersecting $K_{j} \cup \bar{W}_{j-1}$. Then $\bar{W}_{j} \subset U$ by the choice of $k$ and $W_{j}$ is a connected neighborhood of the (connected) set $K_{j} \cup \bar{W}_{j-1}$. The regularity of the sets $W_{j+1} \backslash \bar{W}_{j}$ follows from the fact that open cubes $Q$ are not thin at points $z \in \partial Q$.)

3.2. Theorem. Let $U \subset \mathbb{R}^{d}$ be a bounded domain, $0 \in U$. Then there exists a strictly positive function $w \in \mathscr{C}^{\infty}(U)$ tending to 0 at $\partial U$ such that $\int_{U} h w d \lambda=$ $h(0)$ for all $h \in \mathscr{H}_{\mathrm{b}}(U)$.

Proof. Choose a family $\left(U_{\alpha}\right)_{\alpha>0}$ according to (3.1), and fix a radially symmetric $\mathscr{C}^{\infty}$-function $\tau \geq 0$ on $\mathbb{R}^{d}$ such that $\{\tau>0\}=B_{1}(0)$ and $\int_{\mathbb{R}^{d}} \tau d \lambda=1$. For $y \in U$ put $\rho(y)=\frac{1}{2} \operatorname{dist}(y, \complement U)$ and define

$$
\tau_{y}: x \mapsto(1 / \rho(y))^{d} \tau((x-y) / \rho(y)), \quad x \in \mathbb{R}^{d} .
$$

Of course, $\tau_{y}$ is a $\mathscr{C}^{\infty}$-function on $\mathbb{R}^{d},\{\tau>0\}=B_{\rho(y)}(y)$, and $\int_{\mathbb{R}^{d}} \tau_{y} d \lambda=1$. For every $\alpha>0$ define $\mu_{\alpha}=\varepsilon_{0}^{\mathfrak{C} U_{\alpha}}$ and

$$
w_{\alpha}: x \mapsto \int_{\partial U_{\alpha}} \tau_{y}(x) d \mu_{\alpha}(y), \quad x \in \mathbb{R}^{d} .
$$

Then $w_{\alpha} \in \mathscr{C}^{\infty}(U)$ and $\left\{w_{\alpha}>0\right\}=\bigcup\left\{B_{\rho(y)}(y): y \in \partial U_{\alpha}\right\}$ since $\partial U_{\alpha}$ is the support of $\mu_{\alpha}$. Moreover, $\int_{U} h w_{\alpha} d \lambda=h(0)$ for every $h \in \mathscr{H}_{\mathrm{b}}(U)$ since $\int h d \mu_{\alpha}=h(0)$ and $\tau$ is radially symmetric.

There exists a decreasing sequence $\left(\alpha_{n}\right)$ of strictly positive real numbers such that $\lim _{n \rightarrow \infty} \alpha_{n}=0$ and

$$
B_{\rho(0)}(0) \cup \bigcup_{n=1}^{\infty}\left\{w_{\alpha_{n}}>0\right\}=U
$$

Define

$$
v=\tau_{0}+\sum_{n=1}^{\infty} \frac{1}{2^{n} \sup w_{\alpha_{n}}(U)} w_{\alpha_{n}} .
$$

Obviously $v>0$ on $U$. Given $m \in \mathbb{N}$, there exists a compact subset $K$ of $U$ such that $\tau_{0}=w_{\alpha_{1}}=\cdots=w_{\alpha_{m}}=0$ on $U \backslash K$ and hence $v \leq \sum_{n=m+1}^{\infty} 2^{-n}=$ $2^{-m}$ on $U \backslash K$. Thus $v$ tends to 0 at $\partial U$. Moreover, given $\alpha>0$, there exists $0<\beta<\alpha$ such that $\operatorname{dist}(x, \complement U) \leq \operatorname{dist}\left(x, U_{\alpha}\right)$ for all $x \in U \backslash U_{\beta}$. Taking $m \in \mathbb{N}$ such that $\alpha_{m}<\beta$ we know that, for all $n \geq m, w_{\alpha_{n}}=0$ on $U_{\alpha}$. This shows that $v \in \mathscr{C}^{\infty}(U)$.

If $h \in \mathscr{X}_{b}(U)$ such that $h(0)=0$ then clearly $\int_{U} h v d \lambda=0$. Finally, we define $w=\left(\int_{U} v d \lambda\right)^{-1} v$. Then, for every $h \in \mathscr{H}_{\mathfrak{b}}(U)$,

$$
\int_{U} h w d \lambda=\int_{U}(h-h(0)) w d \lambda+\int_{U} h(0) w d \lambda=0+h(0) \int_{U} w d \lambda=h(0) .
$$


3.3. Corollary. Let $0<\varepsilon<1$, and let $U \subset \mathbb{R}^{d}$ be a bounded domain with $\mathscr{C}^{1+\varepsilon}$-boundary (or, more generally, with Ljapounov-Dini boundary; see [Wi]) such that $0 \in U$. Then there exists a bounded function $w \in \mathscr{C}^{\infty}(U)$ such that inf $w_{0}(U)>0$ and

$$
\int_{U} h w_{0} d \lambda=h(0) \quad \text { for all } h \in \mathscr{H}_{b}(U) .
$$

Proof. Let $g=G_{U}(0, \cdot)$ and

$$
U_{\alpha}=\{g>\alpha\}, \quad \mu_{\alpha}=\varepsilon_{0}^{\complement U_{\alpha}} \quad(0 \leq \alpha<\infty) .
$$

Note that $U_{0}=U$. As proven in [Wi, p. 27] the gradient $\nabla g$ has a continuous extension to $\bar{U}$ (still denoted by $\nabla g$ ) and $\|\nabla g\|$ is bounded away from zero on $\partial U$. Consequently, there exist $c>0$ and $\delta>0$ such that $\|\nabla g\| \geq c$ on $U \backslash U_{\delta}$. Define $w_{1}: U \rightarrow \mathbb{R}$ by

$$
w_{1}= \begin{cases}0 & \text { on } U_{\delta}, \\ \|\nabla g\|^{2} \exp \left(-\frac{1}{\delta-g}\right) & \text { on } U \backslash U_{\delta} .\end{cases}
$$

Then $w_{1} \in \mathscr{C}_{\mathrm{b}}^{\infty}(U)$ and $\inf w_{1}\left(U \backslash U_{\delta / 2}\right)>0$. Let $\sigma$ denote the $(d-1)$ dimensional Hausdorff measure on $\mathbb{R}^{d}$. Recall that, for every $0 \leq \alpha \leq \delta$,

$$
\mu_{\alpha}=\left|\left\langle n_{\alpha}, \nabla g\right\rangle\right| 1_{\partial U_{\alpha}} \sigma=\|\nabla g\| 1_{\partial U_{\alpha}} \sigma
$$

$\left(n_{\alpha}\right.$ exterior normal to $U_{\alpha}$; see [Da]). Fix $h \in \mathscr{H}_{b}(U)$ such that $h(0)=0$. Then

$$
\begin{aligned}
\int_{U} h w_{1} d \lambda & =\int_{U \backslash U_{\delta}} h w_{1} d \lambda \\
& =\int_{0}^{\delta}\left(\int_{\partial U_{\alpha}} h\|\nabla g\|^{2} \exp \left(-\frac{1}{\delta-g}\right) \frac{1}{\mid\left\langle n_{\alpha}, \nabla g\right\rangle} d \sigma\right) d \alpha \\
& =\int_{0}^{\delta}\left(\int_{\partial U_{\alpha}} h \exp \left(-\frac{1}{\delta-\alpha}\right) d \mu_{\alpha}\right) d \alpha \\
& =h(0) \int_{0}^{\delta} \exp \left(-\frac{1}{\delta-\alpha}\right) d \alpha=0 .
\end{aligned}
$$

To finish the proof it now suffices to take a bounded $\mathscr{C}^{\infty}$-function $w_{0}>0$ on $U$ such that $\int_{U} h w_{0} d \lambda=0$ for every $h \in \mathscr{H}_{b}(U)$ with $h(0)=0$ and to define

$$
w=\left(\int_{U}\left(w_{0}+w_{1}\right) d \lambda\right)^{-1}\left(w_{0}+w_{1}\right)
$$

\section{REFERENCES}

[BH] J. Bliedtner and W. Hansen, Potential theory-An analytic and probabilistic approach to balayage, Universitext, Springer, Berlin, 1986.

[CD] G. Choquet and J. Deny, Sur quelques propriétés de moyenne caractéristiques des fonctions harmoniques et polyharmoniques, Bull. Soc. Math. France 72 (1944), 118-140.

[Da] B. E. J. Dahlberg, Estimates of harmonic measure, Arch. Rational Mech. Anal. 65 (1977), 275-288.

[FI] L. Flatto, Fonctions with a mean value property, J. Math. Mech. 10 (1961), 11-18. 
[FL] A. Friedman and W. Littman, Functions satisfying the mean value property, Trans. Amer. Math. Soc. 102 (1962), 167-180.

[Ga] A. M. Garsia, A note on the mean value property, Trans. Amer. Math. Soc. 102 (1962), 181-186.

[Hi] M. R. Hirschfeld, Sur les fonctions $\mu$-harmoniques dans un espace localement compact mesure, C. R. Acad. Sci. Paris Sér. I. Math. 262 (1966), 174-176.

[Sm] E. Smyrnélis, Mesures normales et fonctions harmoniques, Bull. Sci. Math. (2) 95 (1971), 197-207.

[Wi] K.-O. Widman, Inequalities for the Green function and boundary continuity of the gradient of solutions of elliptic differential equations, Math. Scand. 21 (1967), 17-37.

[Za] L. Zalcman, Mean values and differential equations, Israel J. Math. 14 (1973), 339-353.

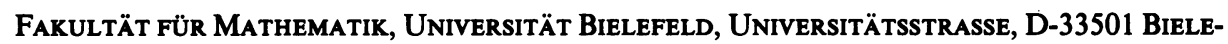
FELD, GERMANY

E-mail address: hansen@mathematik.uni-bielefeld.de

Mathematical Institute, Charles University, Sokolovská 83, 18600 Praha 8, CZech Republic

E-mail address: netuka@karlin.mff.cuni.cz 